\title{
Estudo morfométrico dos ramos intra-hepáticos da artéria hepática na atresia biliar: possívelcontribuição no diagnóstico diferencial entre as formasembriônica e perinatal
}

\author{
João P. U. Fontenele(PG), Vinícius M. Jarry (IC), Cecilia A. F.Escanhoela (PQ)
}

\section{Resumo}

A atresia biliar $(A B)$ é uma causa de icterícia colestática em recém-nascidos cujo diagnóstico continua sendo um desafio. Classicamente duas formas clínicas são reconhecidas, a embriônica e a perinatal, a primeira manifestando-se já ao nascimento e a segunda nas primeiras semanas de vida. Alguns pesquisadores têm descrito o espessamento de ramos intra-hepáticos da artéria hepática como um dos critérios histopatológicos da AB. Avaliamos por técnica de morfometria este dado morfológico em 56 casos de $A B$ e, inicialmente, analisamos estatisticamente 4 casos de apresentação perinatal incomum, com início dos sintomas entre 4 e 29 semanas de vida, e comparamos com um grupo controle.

Palavras Chave:atresia biliar, icterícia neonatal, morfometria.

\section{Introdução}

A atresia biliar $(A B)$ é doença rara que acomete recém-nascidos, podendo se manifestar já ao nascimento ou nas primeiras semanas de vida. Alguns autores têm descrito o espessamento de ramos intra-hepáticos da artéria hepática como critério histopatológico de AB[1]. Nosso objetivo é avaliar a existência deste dado morfológico em pacientes com $A B$ e sua correlação com as formas clínicas embriônica e perinatal.

\section{Resultados e Discussão}

Avaliamos por técnica de morfometria 56 casos de $A B$ tratados com cirurgia de Kasai no Hospital das Clínicas da Unicamp e submetidos à biópsia hepática em cunha transoperatória. Nosso grupo controle $(\mathrm{N}=10)$ foi constituído de espécimes hepáticas provenientes de autópsia do serviço de Anatomia Patológica em pacientes com idade inferior a 1 ano e sem diagnóstico de $A B$. Inicialmente analisamos estatisticamente 4 casos de apresentação perinatal incomum de $A B$ (início de doença entre 4 e 29 semanas de vida). Seguimos metodologia previamente descrita na literatura[2]: estudo imunoistoquímico para camada muscular, captura de imagens e análise morfométrica no programa Imagej1.48a. Foram calculados a espessura da camada média (EM), diâmetro interno(DI) e externo(DE). O grau de espessamento da camada média foi avaliado pelas relações EM/DE x10 e DI/DE x10. Aumento nos valores de EM/DE e/ou diminuição de DI/DE favorecem espessamento. De acordo com o DE os vasos foram classificados como artéria (DE $\geq 100 \mu \mathrm{m}$ ) ou arteríola (DE entre 20 e 100 $\mu \mathrm{m}$ ).
O número total de vasos e médias finais das relações EM/DE e DI/DE dos 4 casos e 10 controles estão expostos na tabela 1.

Tabela 1. Total de vasos e médias finais das relações EM/DE e DI/DE

\begin{tabular}{|c|c|c|c|c|c|c|}
\hline & $\begin{array}{l}\text { Total } \\
\text { Artérias }\end{array}$ & $\begin{array}{l}\text { Total } \\
\text { Arteriolas }\end{array}$ & $\begin{array}{l}\text { Média } \\
\text { EM/DE } \\
\text { Artérias }\end{array}$ & $\begin{array}{l}\text { Média } \\
\text { DUDE } \\
\text { Artérias }\end{array}$ & $\begin{array}{l}\text { Média } \\
\text { EM/DE } \\
\text { Arteriolas }\end{array}$ & $\begin{array}{l}\text { Média } \\
\text { DUDE } \\
\text { Arteriolas }\end{array}$ \\
\hline Casos & 3 & 64 & 1.41 & 7.17 & 1.74 & 6.51 \\
\hline Controles & 10 & 148 & 1,16 & 7.66 & 1.92 & 6.13 \\
\hline
\end{tabular}

Os valores foram analisados pelo teste $U$ de Mann-Whitney no programa GraphPadPrism 5.0 e não foi encontrado variação com significância estatística entre os mesmos $(P<0,05)$.

\section{Conclusões}

Ausência de valores morfométricos compatíveis com espessamento arterial em 4 casos de $A B$ perinatal de apresentação tardia. Análise estatística dos demais casos e formas clínicas em andamento.

\section{Agradecimentos}

\section{Departamento de Anatomia Patológica da FCM}

\footnotetext{
Stowens, D. Congenital BiliaryAtresia. Ann N Y AcadSci1963; 111: 337-357

${ }^{2}$ dos Santos, J.L; da Silveira, T.R; da Silva, V.D;Cerski, C.T; Wagner, M.B. Medial thickeningofhepaticarterybranches in biliaryatresia. A morphometric study. J PediatrSurg2005; 40: 637 642.
} 